\title{
CAMPANHA PRATO LIMPO: EVITANDO O DESPERDÍCIO DE ALIMENTOS NO REFEITÓRIO DO IFPE CAMPUS BELO JARDIM
}

\author{
Silvia Patrícia de Oliveira Silva Bacalhau ${ }^{1}$, Maria do Socorro Santos da Silva ${ }^{1}$, \\ Joelma Fortunato Cordeiro Alves ${ }^{1}$, Francisco das Chagas Lino Lopes ${ }^{1}$, \\ ANDERSON ACIOLI GOMES ${ }^{1}$, Gesilda MARQUES DA SILVA RAMOS ${ }^{1}$, \\ Jessica SABrina de Oliveira Menezes ${ }^{1}$ \\ ${ }^{1}$ Instituto Federal de Educação, Ciência e Tecnologia de Pernambuco (IFPE) - Campus Belo Jardim \\ $<$ michellidaros@gmail.com>,<silvia.oliveira@ reitoria.ifpe.edu.br> \\ <socorro.silva@belojardim.ifpe.edu.br>.<joelma.fortunato@belojardim.ifpe.edu.br> \\ $<\mathrm{dg} @$ belojardim.ifpe.edu.br $>$.<silvinha_pat@ hotmail.com> \\ $<$ gesilda.ramos@belojardim.ifpe.edu.br>. $<$ jessica.oliveira@belojardim.ifpe.edu.br> \\ DOI: $10.21439 /$ conexoes.v10i1.787
}

Resumo. Em comemoração ao Dia Mundial da Alimentação e considerando a quantidade de alimentos desperdiçada no refeitório do Campus Belo Jardim do Instituto Federal de Pernambuco (IFPE), foi lançada em 16 de outubro de 2012 a Campanha Prato Limpo. O objetivo da Campanha foi motivar os usuários a reduzir o desperdício de alimentos, contribuindo para o desafio atual da construção da sustentabilidade da vida no planeta. Este artigo trata-se de um estudo de caso sobre as atividades educativas acerca do tema realizadas durante quatro semanas no refeitório do Campus. As atividades foram compostas de: 1) entrega de folders informativos sobre desperdício de alimentos, alimentação saudável e regras da Campanha; 2) exposição em forma de gêneros alimentícios crus do quantitativo desperdiçado semanalmente: $246 \mathrm{~kg}$; 3) entrega de cartela a ser carimbada sempre que o usuário devolvesse sua bandeja sem restos, para participação de sorteios; 4) concurso de frases e poesias sobre desperdício de alimentos; 5) exposição de frases educativas por todo o refeitório, em português e em inglês; 6) pesagem dos resíduos ao final do horário do almoço. $\mathrm{O}$ concurso de frases teve quatro estudantes vencedores e o concurso de poesia, um. A redução do desperdício chegou a $61 \%$. O objetivo da campanha foi alcançado, porém, é necessária a prática contínua da conscientização dos comensais sobre a importância do combate ao desperdício de alimentos, no sentido de se servirem somente do que vão ingerir. A diminuição na quantidade de resíduos sólidos traz economia para o serviço público e benefícios para o planeta.

Palavras-chaves: Desperdício. Sustentabilidade. Gêneros Alimentícios. Unidade de Alimentação e Nutrição (UAN).

\begin{abstract}
In commemoration at the World Food Day and considering the amount of food wasted in the Campus Belo Jardim's restaurant of Instituto Federal of Pernambuco (IFPE), was released on October 16, 2012 the Clean Dish Campaign. The aim of the Campaign was to motivate users to reduce food waste, contributing to the current challenge of building the sustainability of life on the planet. This article comes up a case study on the educational activities on the subject conducted over four weeks in the campus restaurant. The activities were composed of: 1) delivery of informational brochures about food waste, healthy food and the Campaign rules; 2) exposure in the form of foods raw quantitative wasted week: $542 \mathrm{lb} ; 3$ ) delivery of chart to be stamped whenever if the user give back your tray without remains, to participate in draw; 4) contest phrases and poems about food waste; 5) exposure of educational phrases throughout the restaurant, in Portuguese and English; 6) weighing waste at the end of lunchtime. The contest phrases had four winning students and the poems contest, one. The waste reduction reached $61 \%$. The campaign aim was reached, however, it's necessary the continued practice of awareness of the diners about the importance of combating food waste, in order to serve only what they ingest is required. The decrease in the amount of solid waste brings savings for the public service and benefits for the planet.
\end{abstract}

Keywords: Waste. Sustainability. Foodstuffs. Food and Nutrition Unit (UAN). 


\section{INTRODUÇÃO}

O Brasil é um dos maiores produtores de alimentos do mundo e chega a produzir cerca de $26 \%$ a mais do que necessita para alimentar a sua população. Porém, grande quantidade do que é produzido é desperdiçada, classificando o país entre os dez que mais desperdiçam alimentos no mundo. Entre os fatores mais comuns relacionados ao desperdício de alimentos estão os maus hábitos de preparo e de consumo, que correspondem a aproximadamente $20 \%$ das perdas (INSTITUTO, 2003. FAO, 2012).

Anualmente são descartados na forma de alimentos o equivalente a 26 bilhões de reais, o suficiente para alimentar cerca de 10 milhões de pessoas, ou 2,6 milhões de famílias, o que evidencia a necessidade de campanhas educativas para diminuir o desperdício (GOULART, 2008).

No mundo, há aproximadamente 870 milhões de pessoas que sofrem de subnutrição, segundo a Organização das Nações Unidas para a Alimentação e a Agricultura (FAO) e a fome chega a matar 2,5 milhões de crianças. Uma em cada oito pessoas no mundo estão drasticamente desnutridas. A média de subnutridos representa $12,5 \%$ da população mundial. Os percentuais aumentam para $23,2 \%$ nos países em desenvolvimento e caem para $14,9 \%$ nas nações desenvolvidas (FAO 2012).

Segundo a Pesquisa Nacional por Amostra de Domicílios (PNAD) de 2013, em um total de 2,1 milhões de famílias brasileiras, pelo menos um de seus moradores encontrava-se em situação de insegurança alimentar grave, restrição alimentar na qual pelo menos em uma pessoa da família foi reportada alguma experiência de fome no período investigado (IBGE, 2013).

De acordo com um estudo do Instituto de Pesquisa Econômica Aplicada IPEA cerca de 3,5 milhões de brasileiros saíram da pobreza em 2012. Atualmente, cerca de 15,7 milhões de pessoas vivem na pobreza no Brasil, dos quais 6,5 milhões continuam abaixo da linha de pobreza. Em 2011, esses números eram de 7,6 milhões de pobres e em torno de 19,2 milhões de pessoas na extrema pobreza (IPEA, 2013).

Diariamente, desperdiça-se o equivalente a $39 \mathrm{mil}$ toneladas de alimentos, quantidade suficiente para alimentar 19 milhões de brasileiros, com as três refeições básicas: café da manhã, almoço e jantar (VELLOSO. 2002).

O termo "desperdício"geralmente está atribuído ao conceito de prejuízo e tem sido uma constante preocupação dos gestores, principalmente àqueles de Unidades de Alimentação e Nutrição (UAN) (GONÇALVES et al. 2005).

\begin{abstract}
As Unidades de Alimentação e Nutrição (UAN) são locais destinados à preparação e ao fornecimento de refeições equilibradas, suficientes, completas, harmônicas e adequadas às características e hábitos alimentares da clientela, que contribuam para manter, melhorar ou recuperar a saúde, bem como auxiliar no desenvolvimento de hábitos alimentares saudáveis (CALDAS et al. 2011).
\end{abstract}

Em uma UAN são consideradas sobras os alimentos produzidos e não distribuídos e restos são aquelas quantidades de alimentos devolvidas na bandeja pelo comensal. O controle dos restos é mais difícil de ser realizado que o controle de sobras limpas por envolver a relação comensal-produto, fatores que variam diariamente (VAZ, 2006, HIRSCHBRUCH, 1998). Jogar comida no lixo tem um custo social muito alto e qualquer ação no sentido contrário representa um ato de cidadania. O objetivo deste estudo de caso foi descrever e analisar as informações adquiridas durante uma campanha educativa voltada para motivar os usuários de um refeitório estudantil a reduzir o desperdício de alimentos.

\section{METODOLOGIA}

O Campus Belo Jardim é um dos dezesseis Campi do Instituto Federal de Educação, Ciência e Tecnologia de Pernambuco (IFPE). A Coordenação Geral de Assistência ao Estudante (CGAE), subordinada à Direção de Ensino do Campus (DDE), é responsável por assuntos referentes ao corpo discente, principalmente no que concerne à oferta de qualidade à vivência do estudante em seu percurso formativo. A Assistência ao Estudante age atentamente na promoção de uma educação que visa não somente a formação de um técnico, mas também de um cidadão. A Política de Assistência Estudantil, que norteia as ações da CGAE, possui dentre outros, o Programa Moradia e Refeitório Estudantil, destinado aos estudantes com dificuldades em manter residência/moradia com recursos próprios, especialmente aqueles que residem fora da cidade em que o Campus se localiza. O estudante contemplado pelo referido Programa tem acesso ao refeitório da instituição, onde recebe as três principais refeições e um lanche noturno. O refeitório também serve aos estudantes que possuem compromissos em mais de um turno na instituição, bem como aos docentes, técnicos administrativos e funcionários terceirizados.

No refeitório do Campus Belo Jardim são desperdiçados semanalmente uma média de $246 \mathrm{~kg}$ de alimentos, considerando apenas o horário do almoço. No local são servidos diariamente cerca de 180 desjejuns, 400 almoços e 250 jantas. O cardápio é considerado de ní- 
vel básico e é composto de saladas, duas opções proteicas, guarnição, prato básico (arroz e feijão), sobremesa e/ou suco. A distribuição dos alimentos é realizada de forma parcialmente proporcionada, ou seja, as quantidades das preparações que compõem o cardápio e que o comensal recebe na refeição são servidas por ele mesmo, com exceção do prato proteico, que é servido por funcionário da unidade.

A Campanha Prato Limpo que deu origem a esse estudo de caso foi realizada no refeitório do Campus supracitado e surgiu de uma necessidade de redução dos altos índices de desperdício alimentar detectados no período anterior à mesma. Lançada no Dia Mundial da Alimentação, 16 de outubro de 2012, a campanha teve duração de quatro semanas e as atividades desenvolvidas durante sua vigência foram:

1. distribuição de folders informativos sobre desperdício de alimentos, dados sobre a fome e a miséria no mundo, alimentação saudável e regras para participação da Campanha;

2. exposição de gêneros alimentícios crus sobre uma das mesas na entrada do refeitório. Os gêneros correspondiam ao quantitativo desperdiçado semanalmente. Esse quantitativo foi obtido através da média de peso dos restos alimentares do almoço de três dias não consecutivos;

3. Entrega de cartela a ser carimbada sempre que o usuário devolvesse sua bandeja sem restos de alimentos, para participação de sorteios. Às sextasfeiras, o usuário que somasse os carimbos correspondentes aos cinco dias da semana (segunda a sexta) preenchia o verso da cartela com seus dados para concorrer a prêmios (chocolates, kits de higiene, maquiagem, pen drives, camisas);

4. concurso de frases e poesias sobre desperdício de alimentos. O estudante poderia escrever uma ou mais frases com o tema, sem assinar seu nome. Era permitido informar apenas o número do $\mathrm{CPF}$ abaixo da frase. As frases e a poesia vencedoras foram selecionadas por uma equipe composta de: professora de português do Campus, chefe do Setor de Alimentação e Nutrição (SAN) e nutricionista. Os vencedores foram premiados com camisas e livros;

5. Exposição de cartazes contendo frases sobre o desperdício de alimentos por todo o refeitório, em português e em inglês. As frases foram traduzidas para o inglês através de parceria entre a equipe do SAN, a professora e o monitor da disciplina de Inglês.
6. Pesagem dos resíduos deixados nas bandejas ao final do horário do almoço. Como resíduos, a pesquisa entendeu os restos dos alimentos (considerou ossos e cascas de frutas) depositados em um balde de lixo de 200 litros que se situava na área de devolução de bandejas e utensílios. Ao final do almoço os resíduos eram pesados em uma balança plataforma industrial Filizola, com capacidade de $300 \mathrm{~kg}$, com intervalos de peso de $100 \mathrm{~g}$, onde o peso do balde era descontado.

Para obtenção do valor per capita do desperdício, diariamente o peso do resíduo era dividido pelo número de comensais que frequentaram o refeitório (VAZ. 2006).

Para estimar o número de pessoas que poderiam ser alimentadas com o quantitativo desperdiçado, considerou-se o estudo realizado por Caldas et al. (2011).

\section{RESULTADOS E DISCUSSÃO}

Foram distribuídos cerca de 400 folders informativos sobre desperdício de alimentos, alimentação saudável e regras para participação da Campanha. A exposição de gêneros alimentícios crus mostrou $246 \mathrm{~kg}$ de alimentos sobre uma das mesas na entrada do refeitório, conforme a Figura 1.

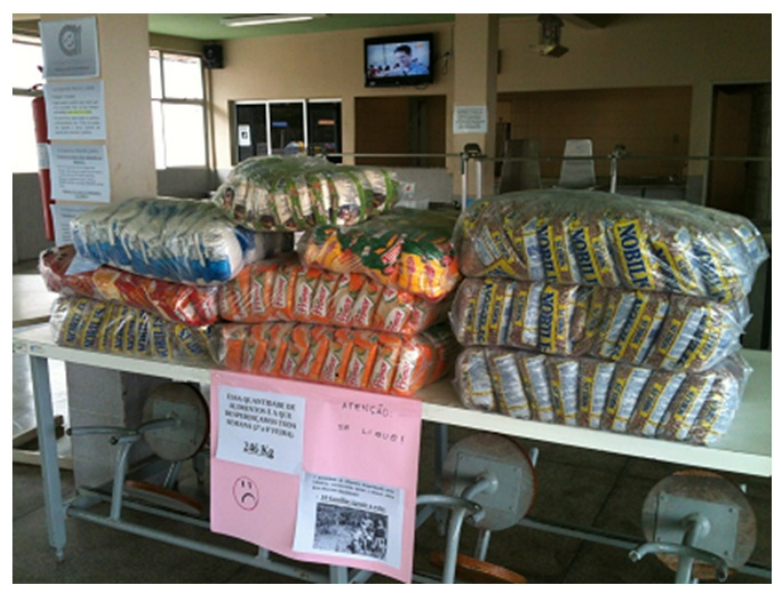

Figura 1: Exposição de $246 \mathrm{~kg}$ de gêneros alimentícios crus na entrada do refeitório do Campus Belo Jardim (IFPE), outubro de 2012. Fonte: Autor

De acordo com a contagem dos cupons participantes dos sorteios ao final de cada semana, cerca de $10 \%$ dos comensais chegavam a completar os cinco espaços do cupom, referentes à entrega da bandeja limpa durante todos os dias da semana, considerando o período de segunda a sexta-feira, conforme a Figura 2 


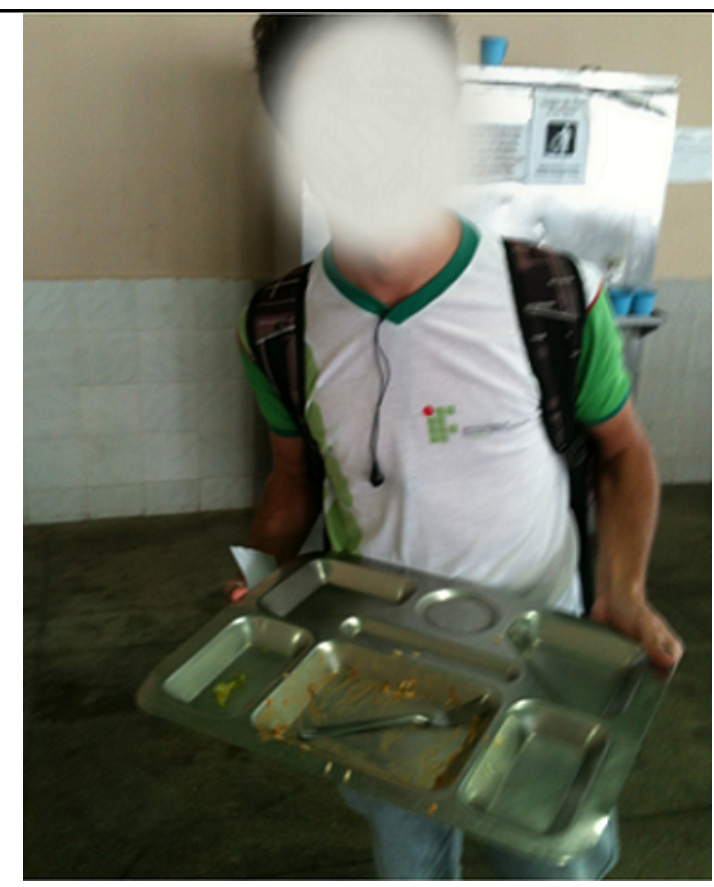

Figura 2: Estudante do Curso de Licenciatura em Música do IFPE Campus Belo Jardim, com sua bandeja limpa, outubro de 2012 Fonte: Autor

O concurso de frases sobre desperdício de alimentos teve como vencedoras:

$1^{\circ}$ LUGAR: "Quem não para pra pensar no desperdício não pensa no próximo."

$2^{\circ}$ LUGAR: "Não desperdice, pois o que você despreza outros veneram..."

$3^{\circ}$ LUGAR (empate): "O desperdício é como o facebook: temos que nos policiar para não ficarmos viciados."e "Abrace a campanha Prato Limpo. Não jogue alimento fora, pois muitas pessoas necessitam desse alimento agora... Diga não ao desperdício!!!"

O concurso de poesias sobre desperdício de alimentos teve como vencedora:

\section{O DESPERDÍCIO}

"A fome é um problema que atinge mais de uma nação.

País pobre, país rico, sem nenhuma exceção, Vamos melhorar, progredir e avançar;

Pois o desperdício não pode continuar;

Entre nessa luta, vamos todos ajudar;

Faça pequenos gestos para a fome erradicar;

Coma bem, coma à vontade,

Mas não coma sem vontade,

Para restos não deixar;

Com este simples gesto,
Você pode nos ajudar,

Para a fome se extinguir,

E o desperdício acabar"

As frases expostas no refeitório, em forma de cartazes, com tradução para o inglês, foram as seguintes:

26,3 milhões de toneladas de alimentos ao ano tem o lixo como destino.

(26,3 million tons of foods have had the trash as destination.)

Diariamente, desperdiçamos o equivalente a 39 mil toneladas de alimentos,

(Every day, we have wasted the equivalent of 39000 tons of foods, )

Quantidade esta suficiente para alimentar $19 \mathrm{mi}-$ lhões de brasileiros, com as três refeições básicas: café da manhã, almoço e jantar.

(These foods have had sufficient to feed 19 million Brazilians, with the three basic meals: breakfast, lunch and dinner.)

A África é o continente caracterizado pela fome e pela miséria.

(The Africa has been characterized as the hunger continent and misery.)

Segundo a ONU, cerca de 150 milhões de pessoas africanas não tem acesso à quantidade mínima de calorias diárias.

(According to the ONU, Approximately, 150 million people in Africa do not have access to the minimum amount of daily calories.)

30 mil crianças morrem de fome a cada dia. (30 thousand children die of hunger every day.)

Há 800 milhões de pessoas desnutridas no mundo. (There are 800 million undernourished people in the world.)

1 bilhão de pessoas passando fome no mundo. (1 billion of people have no food in the world.)

O Brasil é o $9^{\circ}$ país com o maior número de pessoas com fome, tem 15 milhões de crianças desnutridas. (Brazil is the 9th country with the largest number of hungry people and it has 15 million undernourished children.)

Apesar da maioria dos comensais não deixar a bandeja completamente limpa, a pesagem dos resíduos ao 


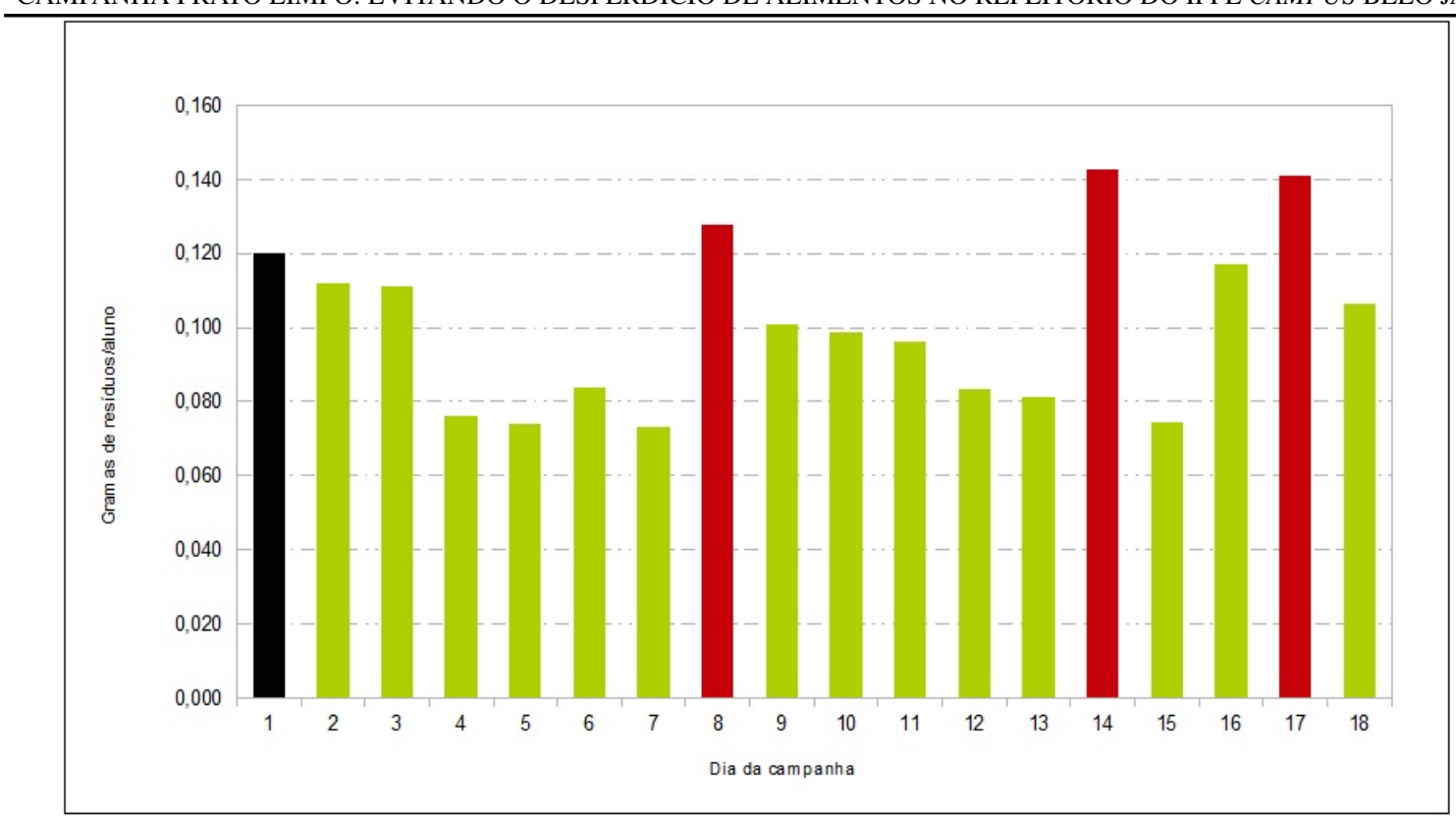

Figura 3: Quantidade média de resíduos per capita mensurada durante a Campanha Prato Limpo realizada no refeitório do IFPE - Campus Belo Jardim, outubro de 2012. Fonte: Autor

final do horário do almoço mostrou uma redução positiva do desperdício, chegando a 60,8\%, como demonstrado no gráfico da Figura 3 . Durante os dezoito dias de duração da campanha, apenas três deles tiveram a quantidade de resíduos superior àquela do primeiro dia de ação e pode-se justificar com o fato de que junto aos restos alimentares foram pesados ossos e cascas de frutas (laranja e melancia), o que tendenciou para mais o quantitativo desperdiçado.

O desperdício na Unidade foi de $567 \mathrm{~kg}$ durante o período da coleta de dados, quantidade que poderia alimentar aproximadamente 909 pessoas, ou 50 pessoas por dia. Uma maneira para reduzir o desperdício seria substituir o bandejão por prato, haja visto que, segundo os participantes de um estudo realizado nos restaurantes do Campus de Ribeirão Preto da Universidade de São Paulo (USP), no prato o comensal se serve menos, no bandejão perde a noção da quantidade (Ferraz Junior, 2010). Augustini et al. (2008) cita que os próprios pratos, sendo estes de tamanho grande, também podem levar os clientes a se servirem de quantidades que não vão consumir.

O Campus Concórdia, do Instituto Federal Catarinense (IFC), já realizou quatro edições de uma campanha semelhante a deste estudo. Com o título de "Bandejão Premiado", foi registrada uma redução de $29 \%$ nas sobras de alimentos(SPERB, 2013).

O desperdício de refeições não é característica apenas do Campus Belo Jardim. Em 2010, um estudo da
Universidade de São Paulo (USP) mapeou o desperdício nos seus restaurantes universitários. A cada semana, cerca de 1,5 tonelada de resíduos eram gerados pelos usuários dos dois restaurantes do Campus de Ribeirão Preto (Ferraz Junior, 2010).

No Instituto Federal de Minas Gerais, Campus Bambuí, a campanha "Desperdício Zero"obteve uma redução de $70 \%$ no desperdício de alimentos ao longo da semana (IFMG, 2014).

Apesar de que no refeitório onde foi realizado o presente estudo os comensais servem-se sós, com exceção do prato proteico, a quantidade de resíduos foi alta. Isso contradiz uma informação referida por um estudo realizado na USP, que cita que com o passar do tempo há uma tendência de acomodação do comensal a se servir apenas da quantidade que sabe que vai comer (Ferraz Junior, 2010).

Apesar dos resultados positivos alcançados, a campanha não atingiu um percentual esperado de estudantes na participação dos sorteios, apenas $10 \%$ dos comensais chegavam a completar os cinco espaços do cupom, referentes à entrega da bandeja limpa todos os dias da semana, considerando de segunda a sexta-feira.

Um fator responsável pelo grande volume de alimentos desperdiçados pode ser de origem cultural, onde o usuário não se importa com a quantidade que se serve nem quanto vai deixar de sobra. Outro fator relacionado ao desperdício pode estar relacionado às preferências alimentares/aceitabilidade das preparações, onde o 
CAMPANHA PRATO LIMPO: EVITANDO O DESPERDÍCIO DE ALIMENTOS NO REFEITÓRIO DO IFPE CAMPUS BELO JARDIM

usuário não gosta de alguma preparação e/ou ingrediente. Os resultados encontrados também podem estar relacionados à temperatura do alimento servido, à questão de que um mesmo prato possa estar sendo preparado com muita frequência, ou que exista uma má aparência ou apresentação das preparações. Os utensílios utilizados para servir também podem interferir, uma vez que podem levar os comensais a se servirem de algo que não irão consumir (AUGUSTINI et al., 2008).

\section{CONSIDERAÇÕES FINAIS}

A Campanha Prato Limpo teve a duração de quatro semanas, porém, é necessária a prática contínua da conscientização dos comensais sobre a importância do combate ao desperdício de alimentos. O grande desafio é conscientizá-los no sentido de servirem-se apenas do que irão ingerir.

Diminuir a quantidade de resíduos sólidos nas instituições de ensino além de trazer economia para o serviço público traz benefícios para o planeta e faz parte do processo educacional do estudante. Um dos maiores desafios para um nutricionista que atende a uma clientela formada por adolescentes em sua maioria é a elaboração de um cardápio saudável, que supra as necessidades nutricionais desse importante estágio da vida, mas que seja bem aceito pelos comensais.

Ações desse cunho também trazem contribuições a nível político-social no desempenho profissional do nutricionista, considerando que o Brasil é um país onde a subnutrição pode ser considerada um sério problema de saúde pública.

\section{AGRADECIMENTOS}

À professora de Língua Portuguesa Jéssica Sabrina, que colaborou fazendo parte do júri para seleção das frases e poesia vencedoras do concurso. Aos funcionários do refeitório do Campus Belo Jardim e aos servidores Geraldo Tavares e Wênia Ventura, pela colaboração durante todo o período da campanha.

\section{REFERÊNCIAS}

AUGUSTINI, V. C. M.; KISHIMOTO, P.; TESCARO, T. C.; ALMEIDA, F. Q. A. Avaliação do índice de resto-ingesta e sobras em unidade de alimentação e nutrição (uan) de uma empresa metalúrgica na cidade de piracicaba / sp. Revista Simbio-Logias, v. 1, n. 1, p. $99-110,2008$.

CALDAS, A. E. C. et al. Avaliação do desperdício de alimentos em unidade de alimentação e nutrição (uan) de um restaurante universitário na cidade de belém - pa. Nutrire, Suplemento $\left(11^{\circ}\right.$ Congresso Nacional da SBAN), n. 36, p. 96-96, 2011.

FAO. FOOD AND AGRICULTURE ORGANIZATION

OF THE UNITED NATIONS. The State of Food

Insecurity in the World 2012. Economic growth is

necessary but not sufficient to accelerate reduction of hunger and malnutrition. Rome, 2012.

Ferraz Junior. Estudo mapeia desperdício em restaurante universitário. 2010. Acesso em: 08 out. 2014. Disponível em: <http: //www.usp.br/agen/?p=42585>

GONÇALVES, B. et al. O compromisso das empresas com o combate ao desperdício de alimentos: Banco de alimentos, colheita urbana e outras ações. São Paulo, 2005 .

GOULART, R. M. M. Desperdício de alimentos: um problema de saúde pública. Integração, n. 54, p.285 - 8. 2008. Acesso em: 14 abr. 2015. Disponível em: $<$ http://www.usjt.br/proex/produtos_academicos/285_ 54.pdf $>$

HIRSCHBRUCH, M. D. Unidades de alimentação e nutrição: desperdício de alimentos x qualidade da produção. Revista Higiene Alimentar, v. 12, n. 55, p. 12 - 14, 1998.

IBGE. Pesquisa Nacional por Amostra de Domicílios PNAD, 2012. Rio de Janeiro, 2013.

IFMG. IFMG - Campus Bambuí promove campanha "Desperdício Zero"em seu Restaurante. 2014. Acesso em: 08 out. 2014. Disponível em: <http://www.cefetbambui.edu.br/portal/ ifmg-campus-bambu-promove-campanhal -desperdicio-zero-em-seu-restaurante>.

INSTITUTO. A nutrição e o consumo consciente. Caderno Temático. São Paulo, 2003. Acesso em: 08 out. 2014. Disponível em: <http://www.akatu.org.br/ Content/Akatu/Arquivos/file/nutricao(2).pdf>

IPEA. Duas décadas de desigualdade e pobreza no Brasil medidas pela Pnad/IBGE, 2013. 159. ed. Brasília, 2013.

RIBEIRO, A. C. M.; SILVA, L. A. Campanha contra o desperdício de alimentos em uma unidade de alimentação e nutrição de curitiba. Revista Brasileira de Nutrição, v. 2, n. 6, p. 329 - 336, 2003.

SPERB, N. C. Redução do desperdício na campanha "Bandejão Premiado". 2013. 
Acesso em: 08 out. 2014. Disponível em:

$<$ http://www.concordia.ifc.edu.br/index.php?

option=com_content\&view=article\&id=631:

reducao-do-desperdicio-na-campanha-bandejao-premiado\&

catid=38:ensino- $\&$ Itemid=132>

VAZ, C. S. Restaurantes - controlando custos e aumentando lucros. Brasília, 2006. 196p.

VELLOSO, R. Comida é o que não falta.

Superinteressante, v. 3, n. 174, p. 01 - 05, 2002. 